\title{
Microelectrophoresis of Silica Rods Using Confocal Microscopy
}

\author{
Henriëtte E. Bakker,*i] Thijs H. Besseling, Judith E. G. J. Wijnhoven, Peter H. Helfferich,
} Alfons van Blaaderen, $*$ and Arnout Imhof*(1)

Soft Condensed Matter, Debye Institute for Nanomaterials Science, Utrecht University, Princetonplein 5, 3584 CC Utrecht, The Netherlands

\section{Supporting Information}

ABSTRACT: The electrophoretic mobility and the zeta potential $(\zeta)$ of fluorescently labeled colloidal silica rods, with an aspect ratio of 3.8 and 6.1, were determined with microelectrophoresis measurements using confocal microscopy. In the case where the colloidal particles all move at the same speed parallel to the direction of the electric field, we record a $x y z$-stack over the whole depth of the capillary. This method is faster and more robust compared to taking xyt-series at different depths inside the capillary to obtain the parabolic flow profile, as was done in previous work from our group. In some

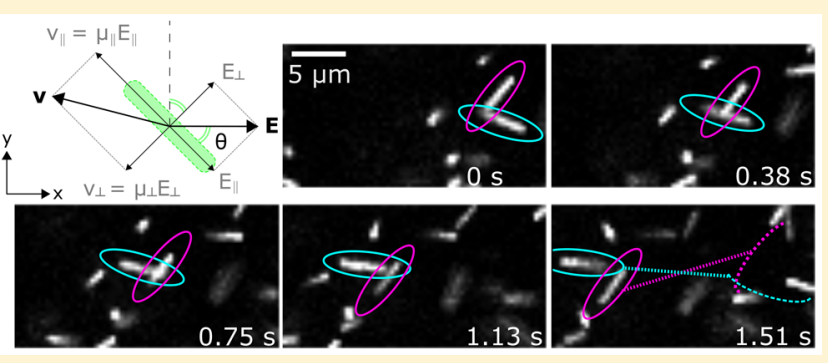
cases, rodlike particles do not move all at the same speed in the electric field, but exhibit a velocity that depends on the angle between the long axis of the rod and the electric field. We measured the orientation-dependent velocity of individual silica rods during electrophoresis as a function of $\kappa a$, where $\kappa^{-1}$ is the double layer thickness and $a$ is the radius of the rod associated with the diameter. Thus, we determined the anisotropic electrophoretic mobility of the silica rods with different sized double layers. The size of the double layer was tuned by suspending silica rods in different solvents at different electrolyte concentrations. We compared these results with theoretical predictions. We show that even at already relatively high $\kappa a$ when the Smoluchowski limiting law is assumed to be valid $(\kappa a>10)$, an orientation dependent velocity was measured. Furthermore, we observed that at decreasing values of $\kappa a$ the anisotropy in the electrophoretic mobility of the rods increases. However, in low polar solvents with $\kappa a<1$, this trend was reversed: the anisotropy in the electrophoretic mobility of the rods decreased. We argue that this decrease is due to end effects, which was already predicted theoretically. When end effects are not taken into account, this will lead to strong underestimation of the experimentally determined zeta potential.

\section{INTRODUCTION}

Charged particle dispersions, with particles ranging from the nano- to micrometer range, are present in everyday life. One could think of small DNA strands, proteins, virusses, micelles, or charged human made colloids, such as those present in e-ink and printer toners. Their surface potential is an important parameter to characterize their stability and interactions. The always present van der Waals forces are attractive if the particles are identical. ${ }^{1}$ In the case where there are no capping molecules present on the particle surface that can provide so-called steric stabilization, $^{2}$ most colloidal particles rely on stabilizing repulsive forces that are caused by excess charges present on/ in the particles. Therefore, measuring electrophoretic mobilities of charged particles is of importance for fundamental studies in a broad range of fields, ${ }^{3-10}$ in biophysics, ${ }^{11-14}$ but also in more applied work in industry. ${ }^{15-19}$

There are fewer papers in which the electrophoretic motion of rodlike particles, the subject of the present paper, is evaluated experimentally in solution, compared to papers for spheres, and most of the published work is from the field of biophysics using anisotropic biomolecules or particles. At relatively thin double layers, $\kappa a \gg 10$, where $\kappa^{-1}$ is the double layer thickness and $a$ is the rod radius, it is well-known that the electrophoretic mobility of a particle does not depend on its shape and it does not rotate in the applied electric field. ${ }^{20}$ For moderate $\kappa a$, Henry already predicted in 1931 that the electrophoretic mobility of infinitely long spherocylinders is dependent on the orientation of the cylinder with respect to the applied electric field. ${ }^{21}$ In general, therefore, a rod moves at an angle with respect to the field. Henry neglected the relaxation effect of the double layer, so that the result is only valid for zeta potentials $(\zeta)$ that are smaller than about $25 \mathrm{mV}\left(\zeta<k_{\mathrm{B}} T / e\right)$. A theory for infinitely long cylinders, where the relaxation effect was included, was developed by Stigter. ${ }^{22,23}$ However, in practice, rodlike particles such as DNA strands, $f d$-virus, microtubules, $f$-actin, or silica rods are finite and need a more accurate description. Using numerical calculations to describe the mobility of finite cylinders, research by both Allison et al. and Buitenhuis, found that with smaller aspect ratio the mobility of spherocylinders decreases at small $\kappa a$, because end effects become more pronounced. ${ }^{24,25}$

Experimentally, most electrophoresis measurements were done using capillairy zone electrophoresis ${ }^{13}$ or electrophoretic light scattering. ${ }^{25,26}$ Using these techniques, only orientationally

Received: October 24, 2016

Revised: December 31, 2016

Published: January 3, 2017 
averaged electrophoretic mobilities of the rodlike particles can be obtained. On the other hand, using optical microscopy, one can measure the electrophoretic mobility of single rodlike particles and its dependence on the orientation with respect to the applied electric field. In this way the mobilities, which in general can be decomposed into two values, a mobility in the direction parallel and perpendicular to the rod, can be measured simultaneously. ${ }^{11,12}$ In addition, the strong sectioning capabilities and increased resolution of confocal microscopy, allow for the investigation of the electrophoresis of concentrated dispersions on the single particle level. ${ }^{3-5}$ Here we make use of the fact that with confocal microscopy it has recently been shown that both coordinates and orientations of rodlike silica model colloids can be obtained as well. ${ }^{27}$ Here, we use silica rods with aspect ratios of 3.8 and 6.1 to measure the anisotropic electrophoretic motion as a function of $\mathrm{Ka}$. Silica rods are well approximated by short and rigid spherocylinders, in contrast to the long and semiflexible microtubules and $f d$ virus used earlier. ${ }^{11,12,23}$ This makes them ideal for use as model particles.

In this paper, we first describe a method to faster measure electrophoretic mobility profiles of colloidal particles using a confocal microscope, as compared to methods used by our group previously. 3 ,4 Measuring electrophoretic mobility profiles, using optical microscopy in closed capillaries, was previously done by measuring $x y t$-series at 10 different depths in the capillary, which is time-consuming. ${ }^{3-5}$ Using crosscorrelation between subsequent frames in a $x y z$-stack, over the whole depth of the capillary, for particles that move all at the same speed parallel to the electric field, we were able to significantly decrease the measurement time needed for microelectrophoresis for rodlike particles in the Smoluchowski limit of high $\kappa a$. This method is also valid for spherical particles. Second, we describe our findings on the anisotropic electrophoretic mobility of colloidal silica rods as a function of the double layer thickness $\kappa a$, by recording $x y t$-series at the stationary plane $z_{\text {stat. }}$. Using confocal microscopy and particle fitting algorithms, ${ }^{27,28}$ we were able to measure the anistropic electrophoretic motion on the single particle level. We dispersed the silica rods in different (mixtures of) solvents of different dielectric constants $\left(\epsilon_{\mathrm{m}}\right)$, which changes both the surface potential of the particles and the double layer thickness, $\kappa^{-1}$. To gain additional control over the double layer thickness in that particular solvent, we also added salt containing a large cation.

We show that, even at already relatively high $\kappa a$, where the Smoluchowski limiting law is often assumed to be valid, an orientation dependent velocity is found. Furthermore, at decreasing values of $\kappa a$ the anisotropy in mobility increases. However, in low polar solvents with lower values of $\kappa a$, the opposite trend was observed. The anisotropy in mobility again decreases. We argue that is due to the fact that end effects start to play a role, which was already predicted theoretically. ${ }^{29}$

\section{EXPERIMENTAL METHODS}

Model System. We used FITC labeled silica rods $\left(\epsilon_{\mathrm{r}} \sim 4.5, n_{\mathrm{D}}^{21}=\right.$ 1.45) that were prepared according to the method of Kuijk and coworkers. $^{30,31}$ Additionally, SR29 rods, which were stable in cyclohexyl chloride (CHC, >98\%, Merck), were modified with octadecyltrimethoxylsilane (OTMOS, $90 \%$, Sigma-Aldrich). ${ }^{32}$ Data on the particles used in this study, can be found in Table 1 and Figure S1. The mean dimensions of the particles and their standard deviations were determined from transmission electron microscopy (TEM) images made with a Philips Tecnai 10 or 12 microscope (Fei Company).
Table 1. Properties of Silica Rods Used in This Study ${ }^{a}$

$\begin{array}{lcccccc} & L, \mathrm{~nm} & \sigma_{\mathrm{L}}, \% & D, \mathrm{~nm} & \sigma_{\mathrm{D}}, \% & L / D & D_{\perp} / D_{\|} \\ \text {R2 } & 3591 & 18 & 587 & 10 & 6.1 & 0.76 \\ \text { SR29 } & 2290 & 6 & 600 & 6.5 & 3.8 & 0.81\end{array}$

${ }^{a} L$ is the end-to-end length of the rod, $D$ is the diameter of the particle, $L / D$ is the aspect ratio of the particle, and $D_{\perp}$ and $D_{\|}$are the estimated translational diffusion coefficient perpendicular and parallel to the rod's long axis, ${ }^{33}$ respectively, calculated from their TEM dimensions.

Typically, 80 particles were measured by hand using the program iTem (Soft Imaging System GmbH, version 5).

For measuring zeta potentials using cross-correlation, the particles were suspended in a refractive index matched solvent mixture, $n_{\mathrm{D}}^{21}=$ 1.45 , of 85 wt $\%$ glycerol ( $\geq 99 \%$, Sigma-Aldrich) and 15 wt \% ultrapure water (Millipore system), with $0.55 \mathrm{mM}$ lithium chloride ( $\mathrm{LiCl})$. For measuring $\mu_{\perp}$ and $\mu_{\|}$of silica rods, the R2 silica rods were dispersed in polar solvents: either 85 wt \% glycerol in ultrapure water or 78 wt $\%$ dimethyl sulfoxide (DMSO, $\geq 99.9 \%$, Sigma-Aldrich) in ultrapure water. $\mathrm{LiCl}$ was added to the more polar solvents to control the thickness of the double layer. SR29 silica rods were dispersed in the nonpolar solvent cyclohexyl chloride (CHC, 98\% Alfa Aesar $\mathrm{GmbH} \& \mathrm{Co} \mathrm{KG}$ ). Tetrabutylammonium chloride (TBAC, $\geq 97 \%$ Sigma-Aldrich) was added to $\mathrm{CHC}$ as a salt to control the double layer thickness. Properties of solvents used in this study can be found in Table 2.

Sample Preparation. For electrophoresis measurements, a sample cell was constructed in the following way. A rectangular borosilicate capillary (Vitrocom, U.K.), with dimensions $(x \times y \times z)$ of either 40 $\mathrm{mm} \times 1 \mathrm{~mm} \times 0.1 \mathrm{~mm}$ or $40 \mathrm{~mm} \times 2 \mathrm{~mm} \times 0.1 \mathrm{~mm}$ was attached to a standard microscope glass slide (Menzel Gläzer) using adhesive tape (Scotch). Two $50 \mu \mathrm{m}$ diameter nickel alloy wires (Good Fellow) were bent into rectangular U-shaped electrodes and inserted in opposite ends of the glass capillary; see Figure $1 \mathrm{~b}$. The typical spacing of the electrodes was $\sim 2 \mathrm{~cm}$. The wires were attached to the glass slide using adhesive tape. The as-prepared electrophoresis cell could only be used once.

The electrophoresis cells were filled with dispersions of silica particles, using a glass Pasteur pipet (Wu Mainz). The dispersions prepared had a volume fraction $\phi \sim 0.01$. It was checked that no air bubbles were present between the electrodes, before sealing the cell with UV-curable glue (Norland Optical adhesive no. 68). The asprepared electrophoresis cells were homogenized in a slowly rotating stage (rotation about the long axis) for 10-60 min before measuring.

Electrophoresis Measurements. The measurements were carried out as follows: a DC-electric field of $1.0-6.3 \mathrm{~V} \mathrm{~mm}^{-1}$ was applied between the electrodes, using a wide band amplifier (KrohnHite, model $7602 \mathrm{M}$ ). The electric field strengths quoted are nominal, that is, based on the applied voltage and the electrode gap. The real electric field inside the cell is typically slightly lower due to electrode polarization and electrode reactions. These potentials are on the order of $1 \mathrm{~V}$, whereas we typically apply a field of $\sim 50 \mathrm{~V}$ with an electrode spacing of $\sim 2 \mathrm{~cm}$. Hence, the difference in nominal field strength used and real electric field strength is only on the order of a few percent. During electrophoresis measurements, we did not observe the occurrence of bubbles near the electrodes due to decomposition reactions such as electrolysis. We believe this is due to the relatively low water content in the solvents used. To record the velocity of the colloids during electrophoresis, we used a laser scanning confocal microscope (Leica TCS SP8) equipped with a $63 \times 1.4 \mathrm{NA}$ oil confocal-objective (Leica). The sample cell was placed with the long axis perpendicular to gravity. To measure electrophoretic mobility profile parallel to gravity (Figure $2 \mathrm{~b}$ ), we used a Nikon $\mathrm{C} 1$ confocal microscope equipped with a $40 \times 1.25 \mathrm{NA}$ oil confocal-objective (Leica) mounted on a microscope body that was tilted $90^{\circ}$. Here, the sample cell was placed with the long axis parallel to gravity, which prevented the formation of a density gradient across the depth $(z)$ of 
Table 2. Properties of Solvents Used in This Study ${ }^{a}$

\begin{tabular}{|c|c|c|c|c|}
\hline & $\rho, \mathrm{g} \mathrm{mL}^{-1}$ & $n_{\mathrm{D}}$ & $\eta, \mathrm{mPa} \mathrm{s}$ & $\epsilon_{\mathrm{m}}$ \\
\hline $\mathrm{CHC}$ & $0.993\left(25^{\circ} \mathrm{C}\right)^{34}$ & $1.46265\left(20^{\circ} \mathrm{C}\right)^{35}$ & $1.5675\left(25^{\circ} \mathrm{C}\right)^{34}$ & $7.6\left(25^{\circ} \mathrm{C}\right)^{36}$ \\
\hline DMSO & $1.096\left(25^{\circ} \mathrm{C}\right)^{37}$ & $1.477\left(25^{\circ} \mathrm{C}\right)^{37}$ & $1.99\left(25^{\circ} \mathrm{C}\right)^{37}$ & $48.4\left(25^{\circ} \mathrm{C}\right)^{38}$ \\
\hline glycerol & $1.258\left(25^{\circ} \mathrm{C}\right)^{39}$ & $1.474\left(20^{\circ} \mathrm{C}\right)^{39}$ & $1410\left(20^{\circ} \mathrm{C}\right)^{39}$ & $41.1\left(20^{\circ} \mathrm{C}\right)^{40}$ \\
\hline $78 \mathrm{wt} \% \mathrm{DMSO} /$ water & $1.096^{*}\left(25^{\circ} \mathrm{C}\right)^{37}$ & $1.45\left(22^{\circ} \mathrm{C}\right)$ & $3.542^{*}\left(25^{\circ} \mathrm{C}\right)^{37}$ & $56.1^{*}\left(25^{\circ} \mathrm{C}\right)^{38}$ \\
\hline $85 \mathrm{wt} \%$ glycerol/water & $1.219\left(25^{\circ} \mathrm{C}\right)^{39}$ & $1.45\left(20^{\circ} \mathrm{C}\right)$ & $81\left(25^{\circ} \mathrm{C}\right)^{41}$ & $49.6^{*}\left(20^{\circ} \mathrm{C}\right)^{40}$ \\
\hline
\end{tabular}

${ }^{a}$ With $\rho$ being the density of the solution, $n_{\mathrm{D}}$ being the refractive index of the solution, $\eta$ being the viscosity of the solution, and $\epsilon_{\mathrm{m}}$ being the dielectric constant of the medium. Values marked with an asterisk $(*)$ were obtained from linear interpolation of literature values.

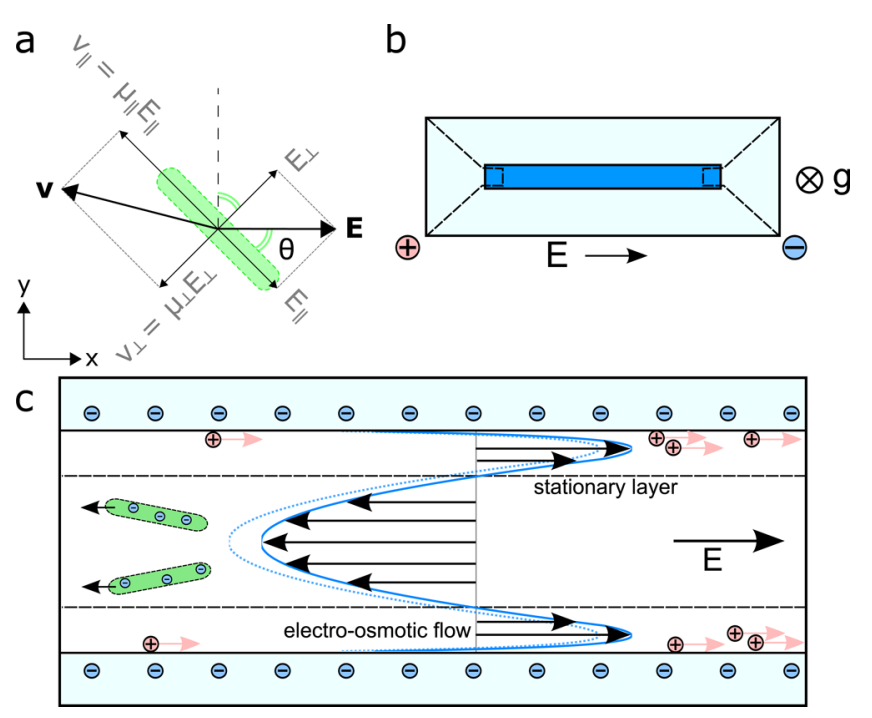

Figure 1. (a) Schematic showing the orientation dependent velocity of a negatively charged spherocylinder subjected to a DC-electric field adapted from Van den Heuvel et al. ${ }^{11}$ (b) Sample cell used during microelectrophoresis. (c) Schematic of flow inside the capillary during microelectrophoresis.

the capillary due to sedimentation of particles that disturbs the recording of mobility profiles.

We recorded series of confocal images in two ways. Either we recorded time series of $x y$-images $(\sim 50-100$ images; typical images size $56 \times 56 \mu \mathrm{m}$; typical pixel size $\sim 0.1-0.3 \mu \mathrm{m}$, time between frames $\Delta t=0.4-1.2 \mathrm{~s}$ ) at fixed depths; typically $\sim 10$ depths over the total height of $0.1 \mathrm{~mm}$ of the electrophoresis cell $(\gtrsim 10 \times 40 \mathrm{~s}) .^{3-5}$ Or we recorded an $x y z$-stack over the whole depth of the capillary (the average step size was $\Delta z \sim 1 \mu \mathrm{m}$ and the time between frames $\Delta t=$ $0.75 \mathrm{~s}$, total measurement time $\sim 75 \mathrm{~s}$ ). The precise positions in $z$ of the top and bottom walls were determined using the reflection mode of the confocal microscope.

Electrophoretic Mobilities. To derive average particle velocities from confocal xyt-series, we took two approaches. In the first approach, we obtained 2D trajectories of particles using particle fitting and tracking algorithms $\mathrm{s}^{27,28}$ to find the average displacement and velocities between frames at height $z$ (xyt particle tracking). Sometimes, the average displacement of particles between two consecutive images was larger than the average distance between colloids, which does not allow for reliable single particle tracking. If this happened, we first used cross-correlation between subsequent frames ${ }^{42}$ to estimate the average displacement, in integer number of pixels, parallel to the electric field ( $x$-direction). After that, the average displacement in $x$ was subtracted from the particle coordinates found. In this "co-moving" frame of reference, the particles could then be tracked. To obtain the true particle trajectories, the average displacement was added back to the $x$-coordinate after particle tracking. The second approach was to use the maximum in the crosscorrelation between two consecutive frames to find the average particle displacement and velocities with subpixel accuracy at height $z^{43}$ (xyt cross-correlation). Next, to derive the average particle velocities from a single $x y z$-stack of the whole depth of the capillary, we used crosscorrelation; cross-correlation of subsequent frames at height $z$ provides the average particle displacement ( $x y z$ cross-correlation). Due to the finite time interval between frames in the stack, particles will appear at shifted positions in subsequent frames.

The electrophoretic mobility of the particles is related to the velocity of the particles, and given by

$$
\mu(z)=v(z) / E
$$

where $v$ is the mean particle velocity $\left(\mathrm{m} \mathrm{s}^{-1}\right)$ and $E$ is the electric field strength $\left(\mathrm{V} \mathrm{m}^{-1}\right)$. During electrophoresis measurements inside a closed capillary, a parabolic Poiseuille flow sets up in the sample cell,
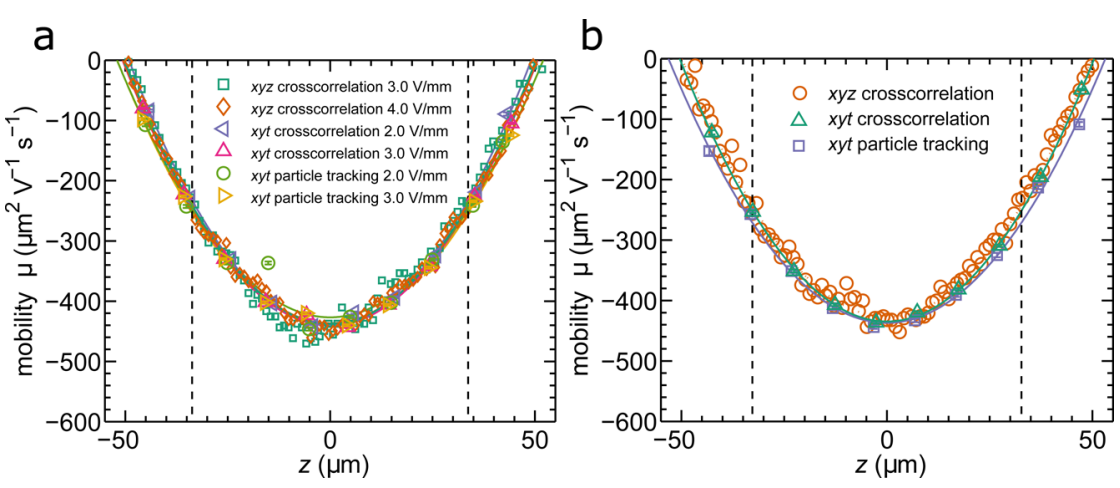

Figure 2. Electrophoretic mobility profiles of R2 rods measured in three different ways. Particles were dispersed in $0.55 \mathrm{mM} \mathrm{LiCl} 85 \mathrm{wt} \% \mathrm{glycerol}-$ water, $\kappa a \approx 24.5$. (a) Measured perpendicular to gravity, $\zeta=-47.0 \pm 0.5 \mathrm{mV}$. (b) Measured parallel to gravity at a field strength of $3 \mathrm{~V} \mathrm{~mm}^{-1}, \zeta=$ $-47.0 \pm 1.6 \mathrm{mV}$. The dashed vertical lines indicate the stationary planes. The lines indicate a parabolic-fit through the data. We set $z=0 \mu \mathrm{m}$ at the maximum of the parabolic profile. Error bars, for xyt cross-correlation and xyt particle tracking, indicate the standard error of the measurement, which is typically smaller than the size of a symbol. The given standard deviation on $\zeta$ is the standard deviation on the three different estimates of $\zeta$ obtained from the parabolic fits through the data. 
due to electro-osmosis of the fluid; ${ }^{44}$ see Figure 1c. Thus, the motion of the particles in the sample cell is a result of the superposition of the electrophoretic velocity and the flow profile setup the by electroosmotic flow (EOF). At the stationary planes, $z=z_{\text {stat }}$, the net flow of the fluid is zero, and the particles solely translate because of their electrophoretic mobility $\mu_{E}=\mu\left(z_{\text {stat }}\right)$ and the superimposed Brownian motion of the particles. In a rectangular cell, the stationary planes $\left(z_{\text {stat }}\right)$ are located at $^{44}$

$$
\frac{z_{\text {stat }}}{h}= \pm \sqrt{\frac{1}{3}+4\left(\frac{2}{\pi}\right)^{5} \frac{1}{k}}
$$

where $k$ is the ratio of the minor and major cross-section of the capillary, $h$ is the distance from the center of the cell $(z=0)$ and either of the walls. We fitted the obtained mobility profiles to a parabolic function, and set $z=0$ at the maximum of the parabolic profile and assumed this to be the center of the capillary. From the value of the fit at $z_{\text {stat }}$ we derived the true electrophoretic mobility. For our capillaries, we measured a depth of $2 h \approx 110 \mu \mathrm{m}$ and $z_{\text {stat }}$ is located at (eq 2) $z_{\text {stat }}$ $\approx \pm 32.7 \mu \mathrm{m}(k \approx 20) \vee \pm 33.7 \mu \mathrm{m}(k \approx 10)$.

In order to determine the anisotropy in mobility $\left(\mu_{\perp} / \mu_{\|}\right)$of our silica rods, we generally recorded only at $z_{\text {stat }}$ image series of typically $\sim 50-100$ frames. An exception was the samples of R2 silica rods dispersed in $85 \mathrm{wt} \%$ glycerol in water; in this case, we took xyt-series at 10 different depths of the sample. The typical images size was $95 \times$ $95 \mu \mathrm{m}$; pixel size $0.37 \mu \mathrm{m}$; the time interval between subsequent frames $\Delta t=0.2-0.9 \mathrm{~s}$.

Solvent Conductivity. An estimate for the Debye screening length, $\kappa^{-1}$, was obtained from the measured conductivities of the solvents used. The conductivities of the polar solvents were measured with a CDM 230 conductivity meter (Radiometer Analytical). The conductivity of $\mathrm{CHC}$, an apolar solvent, was measured using a Scientifica 627 conductivity meter. We assumed that ions contributing to the conductivity in $\mathrm{CHC}$ were $\mathrm{H}^{+}$and $\mathrm{Cl}^{-}$and independent migrations of ions. The conductivity is related to the concentration of dissociated salt, given by.

$$
\sigma=c \Lambda_{0}
$$

$\sigma$ is the measured conductivity $\left(\mathrm{Sm}^{-1}\right), \Lambda_{0}$ is the limiting molar conductance of the salt at infinite solution $\left(\mathrm{Sm}^{2} \mathrm{~mol}^{-1}\right)$ in the given solvent, and $c$ is the concentration of dissociated salt.

Using Walden's rule, one can calculate $\Lambda_{0}$ for different solvents, using literature values of known species. Walden's rule state that the product of the limiting conductance and the viscosity is a constant for the same species in different solvents.

$$
\Lambda_{0}^{A} \eta^{A}=\Lambda_{0}^{B} \eta^{B}
$$

The superscript denotes the particular solvent and $\eta$ is the viscosity (Pa s) of the solvent. In order to calculate $\Lambda_{0}$ for $\mathrm{CHC}$, we used known literature values for $\mathrm{HCl}$ in ethanol, $\Lambda_{0}^{\mathrm{EtOH}}=84.3 \mathrm{~S} \mathrm{~cm}^{2}$ $\mathrm{mol}^{-1},{ }^{45} \eta^{\mathrm{EtOH}}=1.08 \mathrm{mPa}$ s. $^{45}$ For polar solvents, we assumed that ions contributing to the conductivity were $\mathrm{Li}^{+}$and $\mathrm{Cl}^{-}$. In order to estimate $\Lambda_{0}$ in polar solvents, we used literature values for $\mathrm{LiCl}$ in glycerol: $\Lambda_{0}^{\text {glycerol }}=27.1 \times 10^{2} \mathrm{~S} \mathrm{~cm}^{2} \mathrm{~mol}^{-1},{ }^{46} \eta^{\text {glycerol }}=910 \mathrm{mPa} \mathrm{s}(25$ $\left.{ }^{\circ} \mathrm{C}\right) .{ }^{41}$ Literature values for viscosities of the different solvent(mixtures) used can be found in Table 2. Using the relation between the ionic strength and conductivity, we can calculate $\kappa^{-1}$ using the following relation, valid for monovalent ions:

$$
\kappa^{-1}=\sqrt{\frac{\epsilon_{\mathrm{m}} \epsilon_{0} k_{\mathrm{B}} T}{2 N_{\mathrm{A}} c e^{2}}}
$$

with $N_{\mathrm{A}}$ being Avogadro constant and $c$ being the concentration of dissociated monovalent salt in the solvent.

For the polar solvents, either we used solvents with added $\mathrm{LiCl}$ or we used solvents without added salt. In the 85 wt \% glycerol-water mixture, without any salt added, the ionic strength was controlled by the trace ions that are always present. For this mixture, we measured a conductivity of $0.168 \mu \mathrm{S} \mathrm{cm} \mathrm{cm}^{-1}$. Although we do not know the composition of the trace-ions, we used the limiting conductivity for
$\mathrm{LiCl}$ in glycerol to estimate the screening length, which we estimated to be $\kappa^{-1} \sim 32 \mathrm{~nm}$. When $0.55 \mathrm{mM} \mathrm{LiCl}$ was added to the $85 \mathrm{wt} \%$ glycerol-water mixture, all the trace ions in the solution were masked by this salt. For this mixture, we measured a conductivity of $1.13 \mu \mathrm{S}$ $\mathrm{cm}^{-1}$, with a corresponding screening length of $\kappa^{-1} \sim 12 \mathrm{~nm}$. For the 78 wt \% DMSO-water mixture, without any salt added, we measured a conductivity of $0.260 \mu \mathrm{S} \mathrm{cm}^{-1}$, with a corresponding screening length $\kappa^{-1} \sim 132 \mathrm{~nm}$. When $0.067 \mathrm{mM} \mathrm{LiCL}$ was added to the solvent, we measured a conductivity of $1.6 \mu \mathrm{S} \mathrm{cm} \mathrm{cm}^{-1}$, with a corresponding screening length of $\kappa^{-1} \sim 53 \mathrm{~nm}$. For silica rods dispersed in $\mathrm{CHC}$, we aimed for large screening lengths. Therefore, trace ions were removed as much as possible from the solution. ${ }^{47}$ Activated alumina was added to a vial of $\mathrm{CHC}$ that was left on a roller-bench overnight. The cleaned $\mathrm{CHC}$ was then separated from the alumina by centrifugation. Molecular sieves, with a pore size of $4 \AA$, were added to the cleaned $\mathrm{CHC}$. The measured conductivity of the cleaned $\mathrm{CHC}$ was $10 \mathrm{pS} \mathrm{cm}^{-1}$, which corresponds to a screening length of $\kappa^{-1} \sim 7 \mu \mathrm{m}$. Moreover, to decrease the double layer in $\mathrm{CHC}$, also a saturated solution of TBAC in $\mathrm{CHC}$ was made. This was done by adding TBAC to cleaned CHC (up to a concentration of $260 \mathrm{mM}$ ), ${ }^{48}$ and left to equilibrate for 1 week. We then diluted the solvents to $0.26 \mu \mathrm{M}$ and $0.026 \mu \mathrm{M}$. The measured conductivity of $\sim 0.026 \mu \mathrm{M}$ TBAC in CHC was $108 \mathrm{pS}$ $\mathrm{cm}^{-1}$, which corresponds to a screening length $\kappa^{-1} \sim 2.2 \mu \mathrm{m}$. The measured conductivity of $\sim 0.26 \mu \mathrm{M}$ TBAC in $\mathrm{CHC}$ was $4800 \mathrm{pS}$ $\mathrm{cm}^{-1}$, which corresponds to a screening length $\kappa^{-1} \sim 0.33 \mu \mathrm{m}$.

\section{THEORETICAL FRAMEWORK}

The relation between the zeta potential $\zeta$ and electrophoretic mobility $\mu$ is given by

$$
\mu=f(\kappa a, \zeta) \frac{2 \epsilon_{\mathrm{m}} \epsilon_{0} \zeta}{3 \eta}
$$

Here $\epsilon_{\mathrm{m}}$ is the dielectric constant of the solvent, $\epsilon_{0}$ is the permittivity of vacuum, $\eta$ is the viscosity of the medium ( $\mathrm{Pa} \mathrm{s})$, and $f(\kappa a, \zeta)$ is a correction factor that also takes the relaxation effect into account. ${ }^{21-23}$ In the Smoluchowski limit $(\kappa a \gg 1)$, $f(\kappa a, \zeta)$ approaches to $3 / 2$ and this relation is then valid for particles of any shape. ${ }^{20}$

For (infinitely) long rods oriented parallel to the electric field $(\kappa L / 2 \gg 1)$ and ignoring relaxation effects $\left(\zeta<k_{\mathrm{B}} T / e\right)$, the relation between $\zeta$ and $\mu_{\|}$is just the Smoluchowski limiting law: ${ }^{21}$

$$
\mu_{\|}=\frac{\epsilon_{\mathrm{m}} \epsilon_{0} \zeta}{\eta}
$$

For infinitely long rods oriented perpendicular to the electric field, the electrophoretic mobility is given by ${ }^{21-23}$

$$
\mu_{\perp}=f_{\perp}(\kappa a, \zeta) \frac{\epsilon_{\mathrm{m}} \epsilon_{0} \zeta}{\eta}
$$

Approximations of $f_{\perp}(\kappa a, \zeta)$ are depicted in Figure $5,{ }^{49,50}$ and $f_{\perp}(\kappa a)$ approaches 1 at $\kappa a \gg 1$ and $1 / 2$ at $\kappa a \ll 1$.

Hence, for $\kappa a<10^{2}$, the electrophoretic velocity of a cylinder is orientation dependent, such that the rod moves at an angle with respect to the electric field. The experimentally determined anisotropy in mobility can be defined as

$$
f_{\perp}(\kappa a, \zeta)=\mu_{\perp} / \mu_{\|}
$$

Measuring the electrophoretic mobility using scattering techniques, one obtains the orientationally averaged mobility: ${ }^{23,51}$

$$
\mu_{\mathrm{eff}}=\frac{1}{3} \mu_{\|}+\frac{2}{3} \mu_{\perp}
$$


By recording the trajectories and corresponding orientations of rods in an electric field using confocal microscopy, we can obtain $\mu_{\|}$and $\mu_{\perp}$, separately. We determine the particle velocity parallel $v_{x}$ and perpendicular $v_{y}$ to the applied electric field. Figure 1a shows the frame of reference; here the subscripts II and $\perp$ are taken with respect to the rod's long axis. The orientation dependent velocities of the rods in the $x$ and $y$ direction are related to $\mu_{\|}$and $\mu_{\perp},{ }^{11}$ given by

$$
\begin{aligned}
& v_{x}=\left[\left(\mu_{\|}-\mu_{\perp}\right) \cos ^{2} \theta+\left(\mu_{\perp}+\mu_{\mathrm{EOF}}(z)\right)\right] E \\
& v_{y}=\left[\frac{1}{2}\left(\mu_{\|}-\mu_{\perp}\right) \sin 2 \theta\right] E
\end{aligned}
$$

where $\mu_{\mathrm{EOF}}(z)$ is the mobility of the particles due to the electroosmotic flow inside the sample.

As $\mu_{\perp}$ can only be derived from a fit of the data to eq 11 , in practice we derive both $\left(\mu_{\|}-\mu_{\perp}\right)$ and $\mu_{\perp}$ from eq 11 . Using the effective mobility eq 10 , we determine the zeta potential $\zeta$ of the rods

$$
\mu_{\mathrm{eff}}=\frac{\epsilon_{\mathrm{m}} \epsilon_{0} \zeta}{\eta}\left[\frac{1}{3}+\frac{2}{3} f_{\perp}(\kappa a)\right]
$$

\section{RESULTS AND DISCUSSION}

Figure 2 shows the electrophoretic mobility profiles obtained for R2 rods dispersed in a 85 wt \% glycerol-water mixture with $0.55 \mathrm{mM} \mathrm{LiCl}$. In this solvent, $\kappa a \gg 10$ and we assume the Smoluchowski limiting law is valid. Hence, the rods move all at the same speed parallel to the electric field. We measured the electrophoretic mobility profiles perpendicular to gravity and compared the different methods. First, the electrophoretic velocity of the particles was recorded at 10 different depths inside the capillary by recording confocal $x y t$-series. The mean particle mobility at height $z$ was determined either by determining particle trajectories ( $x y t$ particle tracking) or by measuring the mean displacement using cross-correlation between subsequent frames ( $x y t$ cross-correlation). Next, a confocal $x y z$-stack was recorded over the whole depth of the capillary. The mean displacement of particles between subsequent frames in $z$ was determined by using crosscorrelation between subsequent images in the xyz-stack. Hence, a velocity profile of the particles as a function of $z$ inside the sample cell could be obtained from one recorded measurement volume ( $x y z$ cross-correlation). In all three experiments, there were around 60 rods present in every recorded frame. All three methods gave similar electrophoretic mobility profiles and show that the mobilities did not depend on the electric field strength used. This was also the case for the fast $x y z$ cross-correlation method.

When the viscosity $\eta$ of the solvent in which the particles are dispersed is low and the density of the particles does not match the density of the solvent $\left(\rho_{\text {colloid }} \neq \rho_{\text {solvent }}\right)$, sedimentation can occur during the time of the measurement (or creaming for particles with a density lighter than that of the solvent, which is not the case here). For silica particles $>300 \mathrm{~nm}$, that is a concern. Although the sedimentation velocity is small compared to the electrophoretic velocity, accumulation of particles near the bottom can disturb the measured mobility profiles. To prevent significant sedimentation during measurements, the sample cell can be placed with the long axis parallel to gravity and the electrophoretic mobility profile is then measured parallel to gravity. ${ }^{5}$ Figure $2 \mathrm{~b}$ shows the electro- phoretic mobility profiles, measured parallel to gravity, obtained by the same methods as described above. We corrected for the effect of gravity on the apparent mobility of the particles, by taking the average of the two mobility profiles with different field direction, canceling out any effect of gravity. For R2 rods using the Smoluchowski limiting law we found $\zeta=-47.0 \pm 1.6$ $\mathrm{mV}$. This is the same value within the error as was found when the electrophoretic mobility was measured perpendicular to gravity: $\zeta=-47.0 \pm 0.5 \mathrm{mV}$ (Figure $2 \mathrm{a}$ ). We thus found similar results for electrophoretic mobility profiles measured parallel to gravity and mobility profiles measured perpendicular to gravity. Measurement on silica spheres also showed that the three different measurement methods give similar results. ${ }^{5}$

To compare the robustness of the different measurement methods, we calculated the $95 \%$ confidence intervals for the estimated electric mobility from the fit of the different measurement methods using Matlab (version R2016a). We calculated these intervals from data in Figure 2 and from van der Linden et al.; see Table S1. This interval is on average smaller for the $x y z$ cross-correlation method, compared to the $x y t$ particle tracking and $x y t$ cross-correlation method. Thus, the determination of the mobility profile using the $x y z$ crosscorrelation method is the most robust.

To summarize the results thus far, the three different methods to measure electrophoretic mobilities gave similar results. We also found similar results for the electrophoretic mobility profiles measured parallel to gravity and the profiles measured perpendicular to gravity. The fit of the mobility profile for $x y z$ cross-correlation is the more robust, compared to the two other measurement methods. Moreover, the $x y z$ crosscorrelation is much faster $(\sim 75 \mathrm{~s})$ than our earlier measurement technique $x y t$ particle tracking $(\gtrsim 10 \times 40 \mathrm{~s})$. Because $x y z$ cross-correlation is much faster, sedimentation during the measurement is less of a problem. But also the Segre and Silberberg effect or flow focusing ${ }^{52}$ will affect the measurements less. The Segre and Silberberg effect describes that, during laminar Poiseuille flow in a capillary, particles tend to position themselves at $0.6 R$ from the center of the capillary. We observed this unfavorable flow focusing effect during electrophoresis experiments after measuring for $\gtrsim 3$ min. Hence, with $x y z$ cross-correlation one can measure the electrophoretic mobility profile before this effect sets in.

The $x y z$ cross-correlation method of measuring electrophoretic mobility profiles can be applied to both colloidal rods and spheres. However, for rods this is only possible in the limit of high $\kappa a \gg 10$, since the method measures only the mobility component parallel to the field $\left(\mu_{x}\right)$. Because at lower $\kappa a$ rods have an anisotropic mobility that depends on the angle the rods have with respect to the applied electric field (as described above in the Theoretical Framework). In principle, $x y z$ crosscorrelation can still be used at low $\kappa a$ by using eq 13 and assuming infinitely long rods, one can obtain $\zeta$ from the measured $\mu_{\text {eff. }}{ }^{22,50}$ However, measuring both $\mu_{\perp}$ and $\mu_{\|}$to obtain $\zeta$ should be more accurate. The electrophoretic mobility of the $\mathrm{R} 2$ rods was measured at $\kappa a \approx 24.5$, for which we measured an anisotropy in mobility of $\mu_{\perp} / \mu_{\|}=0.98$ (see below). Thus, the rods moved almost parallel to the electric field, and we assumed that the small anisotropy in mobility did not significantly affect the measured $\zeta$. Finally, we wish to remark that the cross-correlation method works best if (longrange) order in the sample is absent, otherwise one will find several peaks in the cross-correlation matrix, making it difficult to determine the real displacement of the sample. 
Anisotropic Electrophoretic Mobilities of Silica Rods. As observed earlier for microtubules, ${ }^{11}$ we observed that the electrophoretic velocity and direction of electrophoretic motion of the colloidal silica rods was in some cases dependent on the orientation of the rods with respect to the electric field; see Figure 1a. This is in line with eqs 11 and 12. A diagram of the electric field components acting on a silica rod that is oriented at an angle $\theta$ with respect to the electric field is shown in Figure 1a. The electric field is along the $x$-axis to the right. The resulting velocity is not parallel to the electric field, but points toward the long axis of the rod, as in general $\mu_{\perp}<\mu_{\|}$.

Figure 3a shows a sequence of cut outs of confocal images of the electrophoretic motion of $\mathrm{R} 2$ silica rods dispersed in a 78
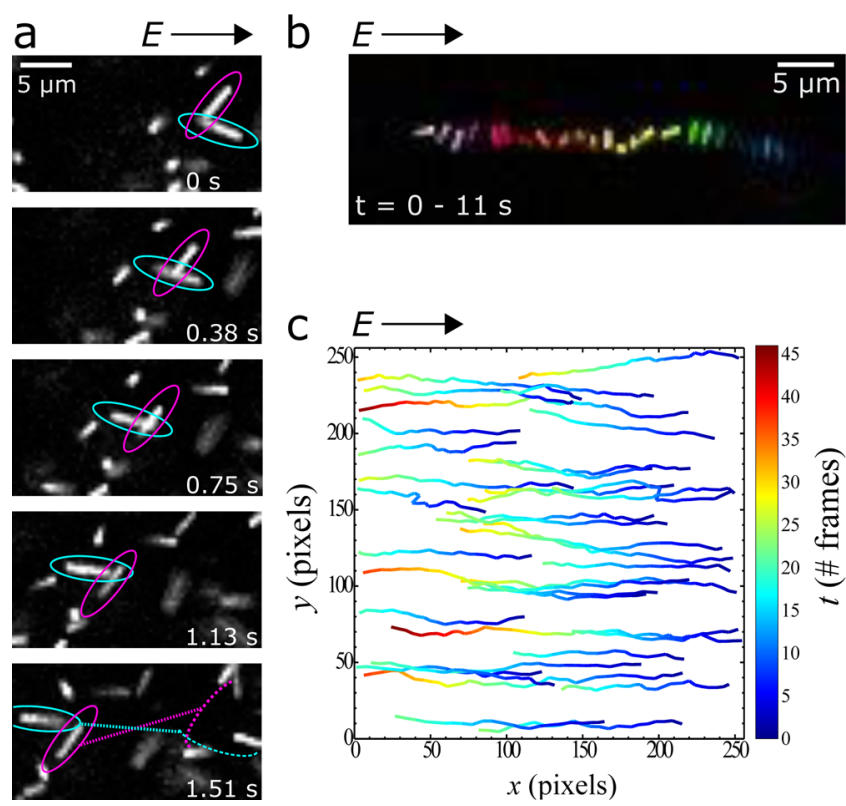

Figure 3. (a) Confocal images from an $x y t$-series of the electrophoresis of R2 silica rods in DMSO--ater $\kappa a=2.2$. Silica rods show an orientation dependent velocity with respect to the applied electric field. (b) Overlay of frames from an $x y t$-series of the electrophoresis of a silica rod dispersed in CHC $\kappa a=0.04,30$ frames, $t=11 \mathrm{~s}, \Delta t=0.37$ $\mathrm{s}, E=2 \mathrm{~V} \mathrm{~mm}^{-1}$. At $t=0$, the color of the rod is blue. The rods still exhibit rotational diffusion, due to Brownian motion. (c) Several trajectories of SR29 rods dispersed in CHC, $\kappa a=0.04$, during electrophoresis. Position of the center of mass is plotted. Minimum length of trajectory displayed here is 20 frames.

wt $\%$ DMSO in water mixture, without any salt added, $\kappa a=2.2$, taken at height $z=z_{\text {stat }}$. In these frames, the rods that are highlighted have different orientations with respect to the electric field. The movement of the rods is not exactly parallel to the electric field, but the velocity has a component in the direction of the long axis of the silica rod; see Supporting Movie 1.

Figure $3 \mathrm{~b}$ shows an overlay of subsequent confocal images taken of the electrophoretic motion of SR29 silica rods in CHC, $\kappa a=0.04$, taken at height $z=z_{\text {stat. }}$ The time interval between the images was $\Delta t=0.37 \mathrm{~s}$. In total, 30 frames were used to make the overlay. At $t=0 \mathrm{~s}$, the color of the rod is blue. The rod moved in a more or less straight line in the direction opposite to the electric field direction, and due to rotational Brownian motion its orientation fluctuated; see Supporting Movie 3.
The orientation dependent velocity of silica rods, dispersed in 78 wt $\%$ DMSO in water, $\kappa a=2.2$, during electrophoresis can be clearly observed by eye. However, for rods dispersed in pure $\mathrm{CHC}, \kappa a=0.04$, it is harder to observe the orientation dependent velocity by eye, because the anisotropy in mobility is smaller.

In order to quantify the exact electrophoretic mobilities of rods orientated parallel and perpendicular to the electric field, we used particle tracking. In each frame we determined the orientations of the rods and recorded their displacements since the previous frame. The displacements were converted to mobilities parallel and perpendicular to the electric field, and were averaged in bins of $4^{\circ}$ in $\theta$. Figure $4 \mathrm{a}$ shows the result for the measured orientation dependent mobility of the R2 silica rods dispersed in 78 wt $\%$ DMSO-water, $\kappa a=2.2$, at $E=1.14$ $\mathrm{V} \mathrm{mm}{ }^{-1}$. Note that the mean square angular displacement, due to the rotational Brownian motion, of the rods in the interval between frames $(0.374 \mathrm{~s})$ is about $12^{\circ}$. The electric field was pointing in the positive $x$-direction. The green symbols indicate the mobility of the rods in the $y$-direction $\mu_{y}$, which is perpendicular to the applied electric field. The red symbols indicate the mobility of the rods in the $x$-direction parallel to the electric field. When the rod was oriented perpendicular or parallel to the electric field $\left(\theta=-90^{\circ} \mathrm{V} 90^{\circ}\right.$ or $\left.\theta=0^{\circ}\right)$, only a mobility component $\mu_{x}$ was observed, parallel to the electric field. For rods that were not completely oriented parallel or perpendicular to the electric field $\left(-90^{\circ}<\theta<0^{\circ}\right.$ or $0^{\circ}<\theta<$ $90^{\circ}$ ), a mobility component perpendicular to the electric field $\mu_{y}$ was found, which was either positive or negative. In other words the rods dispersed in a $78 \mathrm{wt} \% \mathrm{DMSO}-$ water mixture showed an orientation dependent velocity, and depending on the orientation, they also had a velocity component in the positive or negative $y$-direction. The solid lines in Figure $4 \mathrm{a}$ are a fit of eqs 11 and 12 through the unbinned data. From the fitted amplitude $\left(\mu_{\|}-\mu_{\perp}\right)$ and offset $\left(\mu_{\perp}\right)$ of eq 11 , we determined $\mu_{\perp}, \mu_{\|}$, and the anisotropy in mobility $\mu_{\perp} / \mu_{\|}$. In this case, the measured anisotropy was $\mu_{\perp} / \mu_{\|}=0.664 \pm 0.006$. The zeta potential was determined using eqs 9, 10, and 13. In all cases, we used eq 11 to obtain $\left(\mu_{\|}-\mu_{\perp}\right)$, and values obtained from eq 12 were $0-20 \%$ off from the value found by eq 11 . The difference may arise because the fitted values for $\left(\mu_{\|}-\mu_{\perp}\right)$ and $\mu_{\perp}$ in eq 11 are dependent on each other, whereas $\mu_{\perp}$ is not of any influence in eq 12 to determine $\left(\mu_{\|}-\mu_{\perp}\right)$. Figure $4 \mathrm{~b}$ shows the corresponding histogram of displacements of $\mathrm{R} 2$ rods in the $x$ and $y$ direction, respectively parallel or perpendicular to the applied electric field. Because the rods behave Brownian, their orientation fluctuates and hence we find a distribution of displacements (see eqs 11 and 12). Moreover, the main displacement due to the electrophoresis is parallel to electric field, and hence the histogram of displacement in the $x$ direction is centered around a negative displacement.

We measured the orientation-dependent electrophoretic mobilities for silica rods over a range of $\kappa a$. We tuned $\kappa a$ by dispersing the rods in solvents with different dielectric constants, and by the addition of salt to change the concentration of free ions in solutions. Although using different solvents affects $\zeta$, the value of $\mu_{\perp} / \mu_{\|}$is much less affected. The details of the results on the measured orientation dependent mobilities at different $\kappa a$ can be found in the Supporting Information (Figures S2-S7). An overview on these results is presented in Figure 5. The graph shows the anisotropy in mobility of the two types of silica rods $\mu_{\perp} / \mu_{\|}$as a function of $\kappa$. The symbols in the graph are experimental data points of 
a

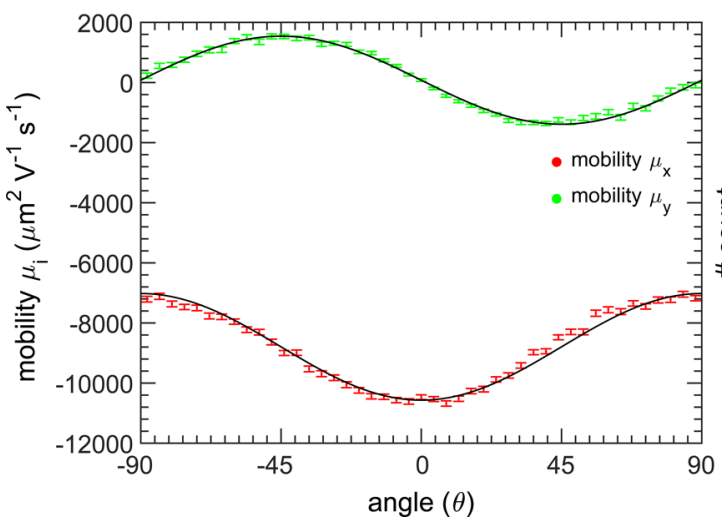

b

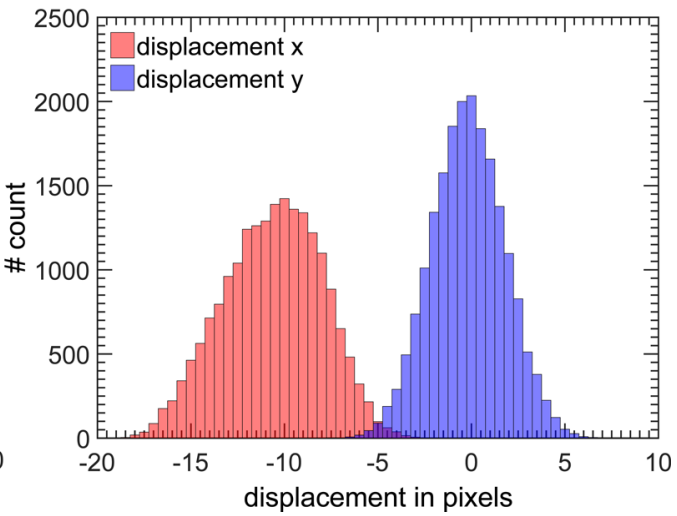

Figure 4. Electrophoresis measurement of $\mathrm{R} 2$ silica rods dispersed in DMSO-water, without salt added, $\kappa \mathrm{a}=2.2$. (a) Orientation dependent mobility in $x$-direction (red symbols), parallel to electric field, and $y$-direction (green symbols), perpendicular to applied electric field. Raw data is binned in $4^{\circ}$ wide bins. The error bars indicate the standard error on the binned data points. The solid lines are a fit through the raw data using eq 11 $\left(\mu_{x}\right)$ and eq $12\left(\mu_{y}\right)$. (b) Histogram of displacements of rods in $x$ and $y$ direction. An anisotropy of $\mu_{\perp} / \mu_{\|}=0.664 \pm 0.006$ was found and $\zeta=-75$ $\mathrm{mV}$. The direction of the electric field was in the positive $x$-direction, $E=1.14 \mathrm{~V} \mathrm{~mm}^{-1}$. $\Delta t=0.374 \mathrm{~s}$. The error on $\mu_{\perp} / \mu_{\|}$is the estimated standard error obtained from the covariance matrix of the fitted parameters.

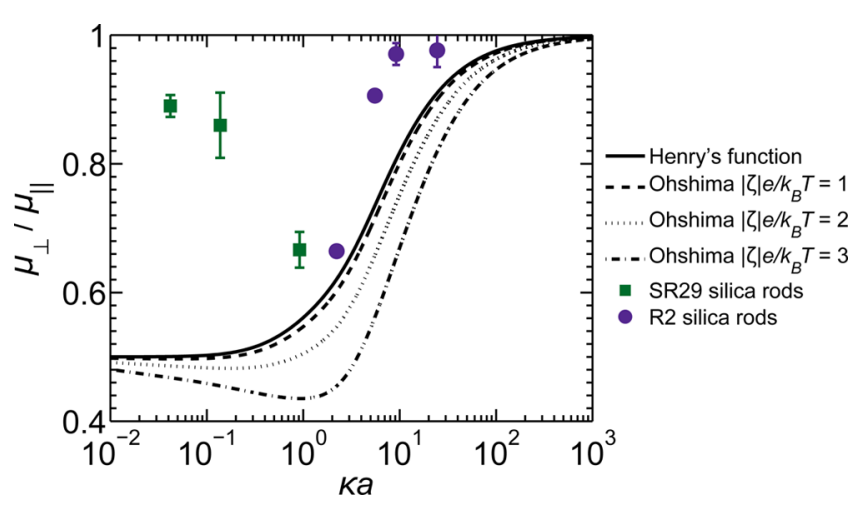

Figure 5. Anisotropy in mobility of silica rods $\left(\mu_{\perp} / \mu_{\|}\right)$plotted as a function of $\kappa a$. The black solid line depicts Ohshima's analytical expression of Henry's solution, for infinitely long rods and low zeta potential $\zeta<k_{\mathrm{B}} T / e^{49}$ The dashed, dotted, and dashed-dotted lines depicts Ohshima's approximate analytical expression for infinitely long rods and moderate zeta potentials, ${ }^{50}$ taking the relaxation effect into account, for $|\zeta| \approx 25 \mathrm{mV},|\zeta| \approx 50 \mathrm{mV}$, and $|\zeta| \approx 75 \mathrm{mV}$, respectively. The symbols are experimental data points of the measured anisotropy in mobility. Squares (green) are SR29 rods in $\mathrm{CHC}$, from left to right: without TBAC added, with $\sim 0.026 \mu \mathrm{M}$, and with $\sim 0.26 \mu \mathrm{M}$ TBAC. Circles (purple) are R2 rods in polar solvents (DMSO-water and glycerol-water), from left to right: $78 \mathrm{wt} \% \mathrm{DMSO}-$ water, $78 \mathrm{wt} \%$ DMSO-water with $\mathrm{LiCl}, 85$ wt \% glycerol-water, and 85 wt \% glycerol-water with $\mathrm{LiCl}$. The error bars indicate the estimated standard deviations.

the measured $\mu_{\perp} / \mu_{\|}$for the SR29 rods in CHC (green squares) and for the R2 rods in polar solvents (purple circles). The black line depicts Ohshima's analytical expression, for Henry's solution of infinitely long rods and low zeta potential $\zeta<$ $k_{\mathrm{B}} T / e^{49}$ The dashed, dotted, and dashed-dotted lines depict Ohshima's approximate analytical expression for infinitely long rods and at moderate zeta potentials, ${ }^{50}$ taking the relaxation effect into account.

For relatively small double layers ( $\mathrm{R} 2$ rods, purple spheres), when $\kappa a>2$, our experimental data show a similar trend as theoretical predictions for infinitely long rods. ${ }^{21,50}$ For SR29 silica rods in apolar solvents with relatively large double layers (green squares), the experimental data do not agree with theoretical predictions for infinitely long cylinders. Rather, $\mu_{\perp} /$ $\mu_{\|}$increases as $\kappa a$ decreases, and the orientation dependent velocity during electrophoresis becomes less pronounced. The difference arises because end effects start to play a role. ${ }^{24,29}$ In this case, the Smoluchowski equation does not hold anymore for rods orientated parallel to an electric field. Calculations on short DNA strands $(\kappa a \sim 1)$ orientated parallel to the electric field, showed that the mobility is significantly reduced compared to longer DNA strands, ${ }^{24}$ while the mobility of rods perpendicular to the electric field remains roughly the same (compared to infinitely long rods). For theoretical calculations on $f d$-virus, a similar trend was observed when the aspect ratio decreased at values of $\kappa a=3.56 \times 10^{-4}$. 25

Because the Smoluchowski limiting law is not valid anymore at $k a<2$ for short rods oriented parallel to the electric field, this would lead to an underestimation of $\zeta$ if we used eq 13. Instead, for $\mu_{\|}$, one should expect a dependence on length, but it is not known analytically. A first estimate is to replace $\mu_{\|}$by a function with the same $\kappa a$ dependence as that for a rod oriented perpendicular to the field, or $f_{\perp}(\kappa L / 2)$. In other words, we expect $\mu_{\perp} / \mu_{\|}=f_{\perp}(\kappa a) / f_{\perp}(\kappa L / 2)$. This is shown in Figure 6 for aspect ratios $L / D=6.1$ and $L / D=3.8$ by using Ohshima's

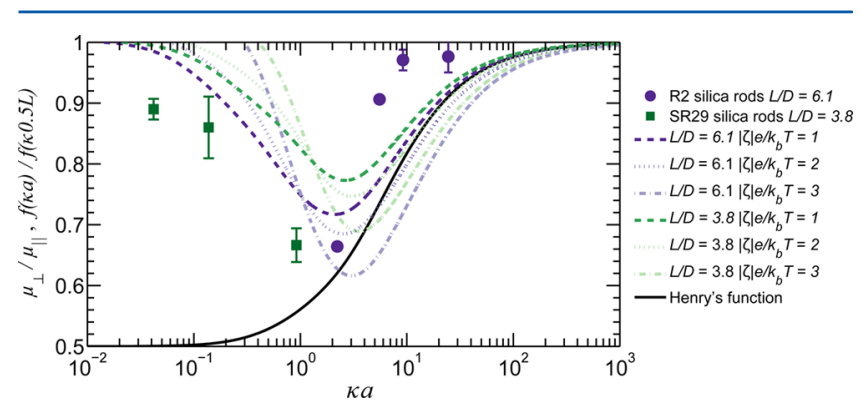

Figure 6. Anisotropy in electrophoretic mobility $\mu_{\perp} / \mu_{\|}$plotted as a function of $\kappa a$. Experimental data from Figure 5 are plotted together with a modified expression for "finite" rods and moderate zeta potentials. This was done by assuming that rods oriented parallel to the electric field are subjected to the same retardation function as rods oriented perpendicular to the electric field: $\mu_{\perp} / \mu_{\|}=f_{\perp}(\kappa a) / f_{\perp}(\kappa L / 2)$. This assumption predicts that for finite rod sizes $\mu_{\perp} / \mu_{\|}$increases as the size of the double layer increases $(\kappa a<1)$. 
approximate analytical expression for infinitely long rods and moderate zeta potentials. ${ }^{50}$ It shows indeed an expected increase of $\mu_{\perp} / \mu_{\|}$at $\kappa a \lesssim 3$. This captures the trend in the experimental data for SR29 rods dispersed in $\mathrm{CHC}$ reasonably.

For $\kappa a>1$, we find larger values of $\mu_{\perp} / \mu_{\|}$than the predictions for infinitely long rods. Although our rods have finite length it may perhaps also partly be attributed to a projection bias. We measured the projected angles of the rods onto the $2 \mathrm{D}$ image plane. This is mainly of influence for rods that appear to have an orientation parallel to the electric the field, as their actual angle with the electric field may be larger. Hence, when these rods would be completely parallel to the electric field, their mobility would be larger. Thus, the actual value of $\mu_{\perp} / \mu_{\|}$is probably smaller than the value we measured.

The anisotropy in mobility in this study was in most cases determined by recording confocal $x y$ t-series only at a height $z=$ $z_{\text {stat }}$ because the rods sediment to the bottom during the electrophoresis measurements before the whole parabolic electro-osmotic flow profile can be measured. SR29 rods dispersed in CHC sediment $100 \mu \mathrm{m}$ in $7 \mathrm{~min}$, the depth of the capillary. R2 rods dispersed in DMSO-water sediment $100 \mu \mathrm{m}$ in $10 \mathrm{~min}$. The drawback of measuring only at $z_{\text {stat }}$ is that $z_{\text {stat }}$ is located in a steep part of the parabolic electro-osmotic flow profile. A small error in the position of $z_{\text {stat }}$ already causes a relatively large error in the determination of $\mu_{\perp}$. Only when the rods were dispersed in a viscous $85 \mathrm{wt} \%$ glycerol-water mixture, the rods did not sediment appreciably during the time scale of the measurements. In this solvent mixture rods take $6 \mathrm{~h}$ to sediment over $100 \mu \mathrm{m}$. In this case, we recorded confocal $x y$-series at 10 different depths inside the capillary; through this determination of the electro-osmotic flow profile, we could more accurately determine $\mu_{\perp}$. To overcome the problem of sedimentation and measure the whole flow profile, a confocal microscope with a tilted frame can be used, ${ }^{5}$ such that the electric field is parallel to gravity. However, due to the relatively long measurement time needed to obtain $\mu_{\perp} / \mu_{\|}$, we encountered other difficulties. At a relatively high volume fraction $(\phi \approx 0.04)$ of rods swirls occurred in the sample, and nonuniform settling was observed. ${ }^{53,54}$ However, when decreasing the volume fraction of rods flow focusing ${ }^{52}$ occurred in the sample within the time scale of the measurement, as described above. Therefore, we determined the anisotropy in mobility at height $z=z_{\text {stat }}$ for rods dispersed in CHC or DMSO-water.

The visual appearance of the motion of silica rods during electrophoresis was dependent on the solvent the silica rods were dispersed in. For instance, in a pure $78 \mathrm{wt} \%$ DMSOwater mixture $(\kappa a=2.2)$ a large anisotropy in mobility of $\mu_{\perp} / \mu_{\|}$ $\sim 0.664$ was measured for the $\mathrm{R} 2$ rods. Hence, the rods showed a clear orientation dependent velocity during electrophoresis. When we look at Supporting Movie 1 of the electrophoretic motion of these silica rods in 78 wt \% DMSO-water, we observed that the silica rods created the impression of "swimming". The direction of motion of the silica rods was approximately in the direction of their long axis. Because the silica rods still behaved Brownian, the angle of each rod with respect to the applied electric field fluctuated, so that the mobility direction changed on a time scale of seconds. The relatively low viscosity ( $3.54 \mathrm{mPa} \mathrm{s})$ of the solvent mixture used in this case results in a calculated rotational diffusion coefficient $D_{\mathrm{r}}=0.032 \mathrm{rad}^{2} \mathrm{~s}^{-1}$. During $1 \mathrm{~s}$, the rods are expected to rotate $\sqrt{4 D_{\mathrm{r}} \tau}=0.35 \mathrm{rad}\left(20^{\circ}\right)$. The combination of Brownian motion and an orientation dependent electrophoretic motion led to an apparent swimming behavior of these silica rods dispersed in DMSO when a DC-electric field was applied to the sample. On the contrary, a sample of silica rods in $85 \mathrm{wt} \%$ glycerol-water showed a completely different behavior (Supporting Movie 2). The silica rods were pulled in a more of less straight line toward the positive electrode, without much rotational diffusion of the rods. This is partly due to the relatively high viscosity of the medium $(81 \mathrm{mPa} \mathrm{s})$ that makes the rotational diffusion of the rods rather slow $D_{\mathrm{r}}=0.0014 \mathrm{rad}^{2} \mathrm{~s}^{-1}$. Furthermore, the measured anisotropy in mobility $\mu_{\perp} / \mu_{\|} \sim 0.97 \mathrm{did}$ not give rise to a clear orientation dependent motion. Supporting Movie 3 then again shows that silica rods in CHC translated at an angle during electrophoresis, without too much "swimming" motion observed because of fast rotational diffusion. In CHC, the SR29 silica rods had a measured anisotropy in mobility $\mu_{\perp} / \mu_{\|} \sim 0.89$. The much lower viscosity $(1.57 \mathrm{mPa} \mathrm{s})$ of $\mathrm{CHC}$, and the slightly smaller dimension of the SR29 silica rods, compared to $\mathrm{R} 2$ rods, results in a higher $D_{\mathrm{r}}=0.195 \mathrm{rad}^{2} \mathrm{~s}^{-1}$ of the rods. Hence, rotational diffusion of the rods was clearly visible on top of the electrophoretic motion the silica rods.

In summary, differences in the observed motions of the silica rods during electrophoresis were due to differences in viscosity and the $\kappa^{-1}$ of the particles due to the solvent the particles were dispersed in. These parameters make for an intriguing interplay between rotational diffusion (Brownian motion) and the orientation dependent electrophoretic mobility.

\section{CONCLUSIONS}

We showed that measuring the electrophoretic mobility, as well as the electro-osmotic flow profile, of colloidal particles that all move at the same speed parallel to the direction of the electric field, can be done by recording an xyz-stack over the whole depth of a capillary using confocal microscopy. Recording a $x y z$-stack is much faster compared to the method that was used previously in which $x y t$-series were recorded at 10 different depths inside the capillary. In the case of rods that did not move all at the same speed through an electric field, we were able to measure the orientation dependent velocity of silica rods during electrophoresis. From this we could determine the anisotropy in the mobility of rods, with an aspect ratio around 5 , during electrophoresis and compare this with theoretical values from literature. We showed that even at relatively high values of $\kappa a$, where the Smoluchowski limiting law was assumed to be valid, a small anisotropy in mobility was found. Furthermore, for the R2 rods dispersed in polar solvent our data agree qualitatively with the theoretical prediction for infinitely long cylinders and low zeta potential $(\kappa a \gtrsim 2)$. However, this theory starts to break down at small values of $\kappa a$, where $\kappa a \lesssim 1$. At these values, end effects start to play a role. Also, the value of the measured anisotropy in mobility was observed to decrease again at smaller values of $\kappa a$. This was already predicted in theoretical calculations for finite cylinders. ${ }^{24,25}$ If this end effect is not taken into account while calculating zeta potentials, this will lead to an underestimation of the experimentally established zeta potential by up to a factor two.

\section{ASSOCIATED CONTENT}

\section{S Supporting Information}

The Supporting Information is available free of charge on the ACS Publications website at DOI: 10.1021/acs.langmuir.6b03863. 
Figures showing TEM images of particles used and the measurements on the orientation dependent electrophoretic mobility of the silica rods in different solvents; table of data on the $95 \%$ confidence interval on the fit for $\mu$ (PDF)

Confocal $x y t$-series of the electrophoresis of $\mathrm{R} 2$ silica rods dispersed in pure 78 wt \% DMSO-water, $\kappa a=2.2$, $\eta=3.542 \mathrm{mPa} \mathrm{s}, \epsilon_{\mathrm{m}}=56.1$; anisotropy of $\mu_{\perp} / \mu_{\|}=0.664$ \pm 0.006 ; time-series shown at $2 \times$ real-time; movie recorded at $z=z_{\text {stat }}$ (AVI)

Confocal $x y t$-series of the electrophoresis of R2 silica rods dispersed in pure 85 wt \% glycerol-water, $\kappa a=9.2$, $\eta=81 \mathrm{mPa} \mathrm{s}, \epsilon_{\mathrm{m}}=49.6$; anisotropy of $\mu_{\perp} / \mu_{\|}=0.97 \pm$ 0.02; time-series shown at $2 \times$ real-time; movie recorded at $z=z_{\text {stat }}$ (AVI)

Cnfocal $x y$-series of the electrophoresis of SR29 silica rods dispersed in deionized $\mathrm{CHC}, \kappa a=0.04, \eta=1.5675$ $\mathrm{mPa} \mathrm{s}, \epsilon_{\mathrm{m}}=7.6$; anisotropy of $\mu_{\perp} / \mu_{\|}=0.89 \pm 0.02$; timeseries shown at $2 \times$ real-time; movie recorded at $z=z_{\text {stat }}$. (AVI)

\section{AUTHOR INFORMATION}

\section{Corresponding Authors}

*E-mail: h.e.bakker1@uu.nl.

*E-mail: a.vanblaaderen@uu.nl.

*E-mail: a.imhof@uu.nl.

ORCID ${ }^{\circ}$

Henriëtte E. Bakker: 0000-0002-6584-6731

Arnout Imhof: 0000-0002-7445-1360

Notes

The authors declare no competing financial interest.

\section{ACKNOWLEDGMENTS}

H.E.B., A.v.B., and A.I. acknowledge financial support from the Foundation for Fundamental Research on Matter (FOM), in the Stirring of Light! program, which is part of The Netherlands Organisation for Scientific Research NWO. H.E.B. and A.v.B. also thank the European Research Council under the European Unions Seventh Framework Programme (FP/2007-2013)/ ERC Grant Agreement No. [291667]: HierarSACol for partial funding of this research. The authors thank Fabian Hagemans for taking the TEM images of Figure S1b.

\section{REFERENCES}

(1) Russel, W. B.; Saville, D. A.; Schowalter, W. R. Colloidal Dispersions, 1st ed.; Cambridge University Press: Cambridge, 1995; p 466.

(2) Napper, D. H. Steric stabilization. J. Colloid Interface Sci. 1977, 58, 390-407.

(3) van der Linden, M. N.; Stiefelhagen, J. C. P.; Heessels-Gürboğa, G.; van der Hoeven, J. E. S.; Elbers, N. A.; Dijkstra, M.; van Blaaderen, A. Charging of poly(methyl methacrylate) (PMMA) colloids in cyclohexyl bromide: Locking, size dependence, and particle mixtures. Langmuir 2015, 31, 65-75.

(4) Vissers, T.; Imhof, A.; Carrique, F.; Delgado, Á V.; van Blaaderen, A. Electrophoresis of concentrated colloidal dispersions in low-polar solvents. J. Colloid Interface Sci. 2011, 361, 443-455.

(5) van der Linden, M. N.; Helfferich, P. H.; Wijnhoven, J. E. G. J.; Bakker, H. E.; van Blaaderen, A. In preparation.

(6) Delgado, A.; González-Caballero, F.; Hunter, R.; Koopal, L.; Lyklema, J. Measurement and interpretation of electrokinetic phenomena. J. Colloid Interface Sci. 2007, 309, 194-224. Elkin 06, International Electrokinetics Conference, June 25-29, Nancy, France.
(7) Pelton, R.; Miller, P.; McPhee, W.; Rajaram, S. Strategies for improving electrophoresis data from the Coulter DELSA. Colloids Surf., A 1993, 80, 181-189.

(8) Yan, D.; Yang, C.; Nguyen, N.-T.; Huang, X. A method for simultaneously determining the zeta potentials of the channel surface and the tracer particles using microparticle image velocimetry technique. Electrophoresis 2006, 27, 620-7.

(9) Yang, C.; Dabros, T.; Li, D.; Czarnecki, J.; Masliyah, J. H. Measurement of the Zeta Potential of Gas Bubbles in Aqueous Solutions by Microelectrophoresis Method. J. Colloid Interface Sci. 2001, 243, 128-135.

(10) Doane, T. L.; Chuang, C.-H.; Hill, R. J.; Burda, C. Nanoparticle $\zeta$-potentials. Acc. Chem. Res. 2012, 45, 317-26.

(11) van den Heuvel, M. G. L.; de Graaff, M. P.; Lemay, S. G.; Dekker, C. Electrophoresis of individual microtubules in microchannels. Proc. Natl. Acad. Sci. U. S. A. 2007, 104, 7770-5.

(12) Li, G.; Wen, Q.; Tang, J. X. Single filament electrophoresis of Factin and filamentous virus fd. J. Chem. Phys. 2005, 122, 104708.

(13) Stellwagen, N. C.; Gelfi, C.; Righetti, P. G. The free solution mobility of DNA. Biopolymers 1997, 42, 687-703.

(14) Strutz, K.; Stellwagen, N. C. Do DNA gel electrophoretic mobilities extrapolate to the free-solution mobility of DNA at zero gel concentration? Electrophoresis 1998, 19, 635-42.

(15) van der Hoeven, P.; Lyklema, J. Electrostatic stabilization in non-aqueous media. Adv. Colloid Interface Sci. 1992, 42, 205-277.

(16) Morrison, I. D. Electrical charges in nonaqueous media. Colloids Surf., A 1993, 71, 1-37.

(17) Gelinck, G. H.; et al. Flexible active-matrix displays and shift registers based on solution-processed organic transistors. Nat. Mater. 2004, 3, 106-10.

(18) Comiskey, B.; Albert, J. D.; Yoshizawa, H.; Jacobson, J. An electrophoretic ink for all-printed reflective electronic displays. Nature 1998, 394, 253-255.

(19) Jenkins, P.; Basu, S.; Keir, R.; Ralston, J.; Thomas, J.; Wolffenbuttel, B. The Electrochemistry of Nonaqueous Copper Phthalocyanine Dispersions in the Presence of a Metal Soap Surfactant: A Simple Equilibrium Site Binding Model. J. Colloid Interface Sci. 1999, 211, 252-263.

(20) Morrison, F. Electrophoresis of a particle of arbitrary shape. J. Colloid Interface Sci. 1970, 34, 210-214.

(21) Henry, D. C. The Cataphoresis of Suspended Particles. Part I. The Equation of Cataphoresis. Proc. R. Soc. London, Ser. A 1931, 133, 106-129.

(22) Stigter, D. Electrophoresis of highly charged colloidal cylinders in univalent salt solutions. 1. Mobility in transverse field. J. Phys. Chem. 1978, 82, 1417-1423.

(23) Stigter, D. Electrophoresis of highly charged colloidal cylinders in univalent salt solutions. 2. Random orientation in external field and application to polyelectrolytes. J. Phys. Chem. 1978, 82, 1424-1429.

(24) Allison, S.; Chen, C.; Stigter, D. The length dependence of translational diffusion, free solution electrophoretic mobility, and electrophoretic tether force of rigid rod-like model duplex DNA. Biophys. J. 2001, 81, 2558-2568.

(25) Buitenhuis, J. Electrophoresis of fd-virus particles: Experiments and an analysis of the effect of finite rod lengths. Langmuir 2012, 28, 13354-13363.

(26) Hoss, U.; Batzill, S.; Deggelmann, M.; Graf, C.; Hagenbuechle, M.; Johner, C.; Kramer, H.; Martin, C.; Overbeck, E.; Weber, R. Electrokinetic Properties of Aqueous Suspensions of Rodlike fd Virus Particles in the Gas- and Liquidlike Phase. Macromolecules 1994, 27, 3429-3431.

(27) Besseling, T. H.; Hermes, M.; Kuijk, A.; de Nijs, B.; Deng, T. S.; Dijkstra, M.; Imhof, A.; van Blaaderen, A. Determination of the positions and orientations of concentrated rod-like colloids from 3D microscopy data. J. Phys.: Condens. Matter 2015, 27, 194109.

(28) Crocker, J.; Grier, D. Methods of Digital Video Microscopy for Colloidal Studies. J. Colloid Interface Sci. 1996, 179, 298-310. 
(29) Allison, S. A.; Mazur, S. Modeling the free solution electrophoretic mobility of short DNA fragments. Biopolymers 1998, 46, 359-373.

(30) Kuijk, A.; Imhof, A.; Verkuijlen, M. H. W.; Besseling, T. H.; van Eck, E. R. H.; van Blaaderen, A. Colloidal Silica Rods: Material Properties and Fluorescent Labeling. Part. Part. Syst. Charact. 2014, 31, 706-713.

(31) Kuijk, A.; van Blaaderen, A.; Imhof, A. Synthesis of monodisperse, rodlike silica colloids with tunable aspect ratio. J. Am. Chem. Soc. 2011, 133, 2346-9.

(32) Liu, B.; Besseling, T. H.; Hermes, M.; Demirörs, A. F.; Imhof, A.; van Blaaderen, A. Switching plastic crystals of colloidal rods with electric fields. Nat. Commun. 2014, 5, 3092.

(33) Tirado, M. M.; Martínez, C. L.; de la Torre, J. G. Comparison of theories for the translational and rotational diffusion coefficients of rod-like macromolecules. Application to short DNA fragments. $J$. Chem. Phys. 1984, 81, 2047.

(34) Rodriguez, S.; Lafuente, C.; Cea, P.; Royo, F. M.; Urieta, J. S. Densities and viscosities of binary mixtures of some cyclic ethers + chlorocyclohexane at 298.15 and 313.15 K. J. Chem. Eng. Data 1997, 42, 1285-1289.

(35) Crowe, R. W.; Smyth, C. P. The Dielectric and Polymorphic Behavior of Cyclohexanol, Cyclohexanone, Chlorocyclohexane and Cyclohexane1,2. J. Am. Chem. Soc. 1951, 73, 5406-5411.

(36) Williams, J. W. The dielectric constants of binary mixtures. X. The electric moments of simple derivatives of cyclohexane and of dioxan. J. Am. Chem. Soc. 1930, 52, 1831-1837.

(37) LeBel, R. G.; Goring, D. A. I. Density, Viscosity, Refractive Index, and Hygroscopicity of Mixtures of Water and Dimethyl Sulfoxide. J. Chem. Eng. Data 1962, 7, 100-101.

(38) Puranik, S. M.; Kumbharkhane, A. C.; Mehrotra, S. C. Dielectric study of dimethyl sulfoxide-water mixtures using the time-domain technique. J. Chem. Soc., Faraday Trans. 1992, 88, 433-435.

(39) Glycerine Producers' Association. Physical properties of glycerine and its solutions; ACI: Washington, D.C., 1967; http://www.aciscience. org/docs/physical_properties_of_glycerine_and_its_solutions.pdf.

(40) Akerlof, G. Dielectric constants of some organic solvent-water mixtures at various temperatures. J. Am. Chem. Soc. 1932, 54, 41254139.

(41) Cheng, N.-S. Formula for the Viscosity of a Glycerol-Water Mixture. Ind. Eng. Chem. Res. 2008, 47, 3285-3288.

(42) Besseling, R.; Isa, L.; Weeks, E. R.; Poon, W. C. K. Quantitative imaging of colloidal flows. Adv. Colloid Interface Sci. 2009, 146, 1-17.

(43) Guizar-Sicairos, M.; Thurman, S. T.; Fienup, J. R. Efficient subpixel image registration algorithms. Opt. Lett. 2008, 33, 156-158.

(44) Hunter, R. J. Zeta Potential in Colloid Science; Elsevier: Amsterdam, 1981.

(45) Janz, G. J.; Danyluk, S. S. Conductances of Hydrogen Halides in Anhydrous Polar Organic Solvents. Chem. Rev. 1960, 60, 209-234.

(46) Benouar, A.; Kameche, M.; Bouhlala, M. A. Molar conductivities of concentrated lithium chloride-glycerol solutions at low and high temperatures: application of a quasi-lattice model. Phys. Chem. Liq. 2016, 54, 62-73.

(47) Vissers, T. Oppositely charged colloids out of equilibrium. Ph.D. Thesis, Utrecht University, The Netherlands, 2010. A digital version of this thesis is available at http://www.colloid.nl.

(48) El Masri, D.; van Oostrum, P.; Smallenburg, F.; Vissers, T.; Imhof, A.; Dijkstra, M.; van Blaaderen, A. Measuring colloidal forces from particle position deviations inside an optical trap. Soft Matter 2011, 7, 3462-3466.

(49) Ohshima, H. Henry's Function for Electrophoresis of a Cylindrical Colloidal Particle. J. Colloid Interface Sci. 1996, 180, 299-301.

(50) Ohshima, H. Approximate Analytic Expression for the Electrophoretic Mobility of Moderately Charged Cylindrical Colloidal Particles. Langmuir 2015, 31, 13633-8.

(51) de Keizer, A.; van der Drift, W.; Overbeek, J. Electrophoresis of randomly oriented cylindrical particles. Biophys. Chem. 1975, 3, 107108.
(52) Segré, G.; Silberberg, a. Behaviour of macroscopic rigid spheres in Poiseuille flow Part 2. Experimental results and interpretation. J. Fluid Mech. 1962, 14, 136.

(53) Royall, C. P.; Dzubiella, J.; Schmidt, M.; van Blaaderen, A. Nonequilibrium sedimentation of colloids on the particle scale. Phys. Rev. Lett. 2007, 98, 188304 DOI: 10.1103/PhysRevLett.98.188304.

(54) Wysocki, A.; Royall, C. P.; Winkler, R. G.; Gompper, G.; Tanaka, H.; van Blaaderen, A.; Löwen, H. Direct observation of hydrodynamic instabilities in a driven non-uniform colloidal dispersion. Soft Matter 2009, 5, 1340. 